TRANSACTIONS OF THE

AMERICAN MATHEMATICAL SOCIETY

Volume 364, Number 7, July 2012, Pages 3349-3363

S 0002-9947(2012)05620-0

Article electronically published on March 7, 2012

\title{
THE DIAMETER ESTIMATE AND ITS APPLICATION TO CR OBATA'S THEOREM \\ ON CLOSED PSEUDOHERMITIAN $(2 n+1)$-MANIFOLDS
}

\author{
SHU-CHENG CHANG AND CHIN-TUNG WU
}

\begin{abstract}
In this paper, we obtain a sharp lower bound estimate for diameters with respect to an adapted metric in closed pseudohermitian $(2 n+1)$ manifolds when a sharp lower bound estimate for the first positive eigenvalue of the sublaplacian is achieved. As a consequence, we confirm the CR Obata Conjecture on a closed pseudohermitian $(2 n+1)$-manifold with an extra condition on covariant derivatives of torsion.
\end{abstract}

\section{INTRODUCTION}

Let $(M, J, \theta)$ be a closed pseudohermitian $(2 n+1)$-manifold (see the next section for basic notions in pseudohermitian geometry). A. Greenleaf ( $\mathrm{Gr}]$ ) proved the pseudohermitian analogue of Lichnerowicz's Theorem ([L]) for the lower bound of the first positive eigenvalue $\lambda_{1}$ of the sublaplacian for a pseudohermitian manifold $M^{2 n+1}$ with $n \geq 3$. In LL, S.-Y. Li and H.-S. Luk proved the same result for the cases $n=2$ and $n=1$ with an extra condition on a covariant derivative of the pseudohermitian torsion.

Recently, it was proved by H.-L. Chiu and the first author ([Chi], CC1] ) that

$$
\lambda_{1} \geq \frac{n k}{n+1}
$$

if

$$
\operatorname{Ric}_{m}(Z, Z)-\frac{n+1}{2} \operatorname{Tor}_{m}(Z, Z) \geq k\langle Z, Z\rangle_{L_{\theta}},
$$

for all $m \in M, Z \in T_{1,0}$, and for some positive constant $k$, on a closed pseudohermitian $(2 n+1)$-manifold with the nonnegative CR Paneitz operator $P_{0}$ if $n=1$.

It is well known that

$$
\lambda_{1}=\frac{n k}{n+1}
$$

on the standard unit sphere $\left(S^{2 n+1}, \widehat{J}, \widehat{\theta}\right)$ in $\mathbb{C}^{n+1}$ with

$$
\operatorname{Ric}_{m}(Z, Z)=k\langle Z, Z\rangle_{L_{\theta}} .
$$

Then it is natural to conjecture the CR analogue of M. Obata's Theorem ([ $)$ on a closed pseudohermitian $(2 n+1)$-manifold $(M, J, \theta)$.

Received by the editors February 3, 2010.

2010 Mathematics Subject Classification. Primary 32V05, 32V20; Secondary 53C56.

Key words and phrases. Lichnerowicz-Obata Theorem, sublaplacian, CR Paneitz operator, pseudohermitian manifold, pseudohermitian Ricci curvature, pseudohermitian torsion.

This research was supported in part by NSC. 
Conjecture 1.1. Let $(M, J, \theta)$ be a closed pseudohermitian $(2 n+1)$-manifold. In addition we assume the Paneitz operator $P_{0}$ is nonnegative if $n=1$. Suppose that

$$
\operatorname{Ric}_{m}(Z, Z)-\frac{n+1}{2} \operatorname{Tor}_{m}(Z, Z) \geq k\langle Z, Z\rangle_{L_{\theta}},
$$

for all $m \in M, Z \in T_{1,0}$, and for some positive constant $k$. Assume that

$$
\lambda_{1}=\frac{n k}{n+1} .
$$

Then $(M, J, \theta)$ is the standard pseudohermitian $(2 n+1)$-sphere $\left(S^{2 n+1}, \widehat{J}, \widehat{\theta}\right)$ with $\operatorname{Ric}_{m}(Z, Z)=k\langle Z, Z\rangle_{L_{\theta}}$.

We first recall some basic definitions.

Definition 1.1. A piecewise smooth curve $\gamma:[0,1] \rightarrow M$ is said to be horizontal if $\gamma^{\prime}(t) \in \xi$ whenever $\gamma^{\prime}(t)$ exists. The length of $\gamma$ is then defined by

$$
l(\gamma)=\int_{0}^{1}\left\langle\gamma^{\prime}(t), \gamma^{\prime}(t)\right\rangle_{L_{\theta}}^{\frac{1}{2}} d t
$$

The Carnot-Carathéodory distance between two points $p, q \in M$ is

$$
d_{c}(p, q)=\inf \left\{l(\gamma) \mid \gamma \in C_{p, q}\right\},
$$

where $C_{p, q}$ is the set of all horizontal curves joining $p$ and $q$. By the Chow connectivity theorem [Cho, there always exists a horizontal curve joining $p$ and $q$, so the distance is finite. The diameter $d_{c}$ is defined by

$$
d_{c}(M)=\sup \left\{d_{c}(p, q) \mid p, q \in M\right\} .
$$

Note that there is a minimizing geodesic joining $p$ and $q$ so that its length is equal to the distance $d_{c}(p, q)$.

Definition 1.2. Let $(M, J, \theta)$ be a closed pseudohermitian $(2 n+1)$-manifold. Define

$$
P \varphi=\sum_{\alpha=1}^{n}\left(\varphi_{\bar{\alpha}}{ }^{\bar{\alpha}}{ }_{\beta}+i n A_{\beta \alpha} \varphi^{\alpha}\right) \theta^{\beta}=\left(P_{\beta} \varphi\right) \theta^{\beta}, \quad \beta=1,2, \cdots, n,
$$

which is an operator that characterizes CR-pluriharmonic functions ( $\mathrm{Le}$ for $n=1$ and GL for $n \geq 2$ ). Here

$$
P_{\beta} \varphi=\sum_{\alpha=1}^{n}\left(\varphi_{\bar{\alpha}}{ }^{\bar{\alpha}}{ }_{\beta}+i n A_{\beta \alpha} \varphi^{\alpha}\right)
$$

and $\bar{P} \varphi=\left(\bar{P}_{\beta} \varphi\right) \theta^{\bar{\beta}}$, the conjugate of $P$. Moreover, we define

$$
P_{0} \varphi=4\left(\delta_{b}(P \varphi)+\bar{\delta}_{b}(\bar{P} \varphi)\right),
$$

which is the so-called CR Paneitz operator $P_{0}$. Here $\delta_{b}$ is the divergence operator that takes $(1,0)$-forms to functions by $\delta_{b}\left(\sigma_{\alpha} \theta^{\alpha}\right)=\sigma_{\alpha}{ }^{\alpha}$ and $\bar{\delta}_{b}\left(\sigma_{\bar{\alpha}} \theta^{\bar{\alpha}}\right)=\sigma_{\bar{\alpha}}{ }^{\bar{\alpha}}$. If we define $\partial_{b} \varphi=\varphi_{\alpha} \theta^{\alpha}$ and $\bar{\partial}_{b} \varphi=\varphi_{\bar{\alpha}} \theta^{\bar{\alpha}}$, then the formal adjoint of $\partial_{b}$ on functions (with respect to the Levi form and the volume form $d \mu$ ) is $\partial_{b}^{*}=-\delta_{b}$. We observe that

$$
\int\left\langle P \varphi+\bar{P} \varphi, d_{b} \varphi\right\rangle_{L_{\theta}^{*}} d \mu=-\frac{1}{4} \int P_{0} \varphi \cdot \varphi d \mu .
$$

For the details about these operators, the reader can make reference to GL and Hi]. 
Definition 1.3. On a closed pseudohermitian $(2 n+1)$-manifold $(M, J, \theta)$, we call the Paneitz operator $P_{0}$ with respect to $(J, \theta)$ nonnegative if

$$
\int_{M} P_{0} \varphi \cdot \varphi d \mu \geq 0
$$

for all real $C^{\infty}$ smooth functions $\varphi$.

Remark 1.1. (i) Let $(M, J, \theta)$ be a closed pseudohermitian $(2 n+1)$-manifold with $n \geq 2$. Then a smooth real-valued function $\varphi$ satisfies $P_{0} \varphi=0$ on $M$ if and only if $P_{\beta} \varphi=0$ on $M$ for all $\beta$. It also holds for a closed pseudohermitian 3-manifold with vanishing torsion ([Hi], GL]).

(ii) Let $(M, J, \theta)$ be a closed pseudohermitian 3-manifold with vanishing torsion. Then the corresponding CR Paneitz operator is nonnegative. Unlike $n=1$, let $(M, J, \theta)$ be a closed pseudohermitian $(2 n+1)$-manifold with $n \geq 2$. The corresponding CR Paneitz operator is always nonnegative ([CCC], [CC2 ]).

Definition $1.4(\mathrm{GL})$. Let $(M, J, \theta)$ be a closed pseudohermitian $(2 n+1)$-manifold. We define the purely holomorphic second-order operator $Q$ by

$$
Q \varphi=2 i\left(A^{\alpha \beta} \varphi_{\alpha}\right)_{\beta} .
$$

Note that $\left[\Delta_{b}, T\right]=2 \operatorname{Im} Q$ and $P_{0}=2\left(\Delta_{b}^{2}+n^{2} T^{2}-2 n \operatorname{Re} Q\right)$.

Definition 1.5. Let $(M, J, \theta)$ be a closed pseudohermitian $(2 n+1)$-manifold. We can define a family of Webster (adapted) Riemannian metrics $g_{\varepsilon}$ of $(M, J, \theta)$ by

$$
g_{\varepsilon}=d \theta+\varepsilon^{-2} \theta^{2}, \varepsilon>0 .
$$

We first obtain the following lower bound estimate of diameter $d_{\varepsilon}(M)$ with respect to an adapted metric $g_{\varepsilon}$.

Proposition 1.2. Let $(M, J, \theta)$ be a closed pseudohermitian $(2 n+1)$-manifold. In addition we assume that the Paneitz operator $P_{0}$ is nonnegative if $n=1$. Suppose that

and

$$
\operatorname{Ric}_{m}(Z, Z)-\frac{n+1}{2} \operatorname{Tor}_{m}(Z, Z) \geq k\langle Z, Z\rangle_{L_{\theta}}
$$

for all $m \in M, Z \in T_{1,0}$, some positive constant $k$ and

$$
\left[\operatorname{Tor}_{m}+\frac{4(n+1)}{k} A^{2}\right](Z, Z) \geq 0
$$

$$
A_{\alpha \gamma, \bar{\gamma}}=0 \quad \text { for all } \alpha \text {. }
$$

Assume that

$$
\lambda_{1}=\frac{n k}{n+1} .
$$

Then

$$
d_{\varepsilon}(M) \geq \sqrt{\frac{n+1}{k}} \pi \quad \text { with } \quad \frac{n+1}{k}=\varepsilon^{2} .
$$

Remark 1.2. (i) In a closed pseudohermitian $(2 n+1)$-manifold with $n \geq 2$, the corresponding condition

$$
P_{\beta} \varphi=0
$$

is always satisfied under $\operatorname{Ric}_{m}(Z, Z)-\frac{n+1}{2} \operatorname{Tor}_{m}(Z, Z) \geq k\langle Z, Z\rangle_{L_{\theta}}$ and $\lambda_{1}=\frac{n k}{n+1}$. It is not true in the case of $n=1$. 
(ii) Note that for $n=1$,

$$
\left[\text { Tor }+\frac{8}{k} A^{2}\right]\left(\left(\nabla_{b} \varphi\right)_{\mathbb{C}},\left(\nabla_{b} \varphi\right)_{\mathbb{C}}\right)=2 \operatorname{Im}\left(A_{11} \varphi_{\overline{1}} \varphi_{\overline{1}}\right)+\frac{4}{k}\left|A_{11}\right|^{2}\left|\nabla_{b} \varphi\right|^{2} .
$$

Then from Lemma 3.4 condition (1.2) will be

$$
\left[\text { Tor }+\frac{8}{k} A^{2}\right]\left(\left(\nabla_{b} \varphi\right)_{\mathbb{C}},\left(\nabla_{b} \varphi\right)_{\mathbb{C}}\right)=0
$$

if $P_{1} \varphi=0$ for the corresponding eigenfunction $\varphi$.

(iii) For $n=1$, if $\operatorname{Ric}_{m}(Z, Z) \geq k\langle Z, Z\rangle_{L_{\theta}}$ and $\lambda_{1}=\frac{k}{2}$ with $A_{11}=0$, then from Lemma 3.2

and from Remark 1.1

$$
P_{0} \varphi=0
$$

$$
P_{1} \varphi=0 .
$$

In the present paper, via the diameter estimate $d_{\varepsilon}(M)$ with respect to the Webster metric $g_{\varepsilon}$, we confirm Conjecture 1.1 in a closed pseudohermitian $(2 n+1)$ manifold with an extra condition on covariant derivatives of torsion.

Theorem 1.3. Let $(M, J, \theta)$ be a closed pseudohermitian $(2 n+1)$-manifold with $n \geq 2$. Suppose that

$$
\operatorname{Ric}_{m}(Z, Z)-\frac{n+1}{2} \operatorname{Tor}_{m}(Z, Z) \geq k\langle Z, Z\rangle_{L_{\theta}},
$$

for all $m \in M, Z \in T_{1,0}$, some positive constant $k$ and

$$
A_{\alpha \gamma, \bar{\gamma}}=0 ; \quad A_{\alpha \beta, \gamma \bar{\gamma}}=0 \text { for all } \alpha, \beta \text {. }
$$

Assume that

$$
\lambda_{1}=\frac{n k}{n+1} .
$$

Then

$$
d_{\varepsilon}(M)=\sqrt{\frac{n+1}{k}} \pi \text { with } \frac{n+1}{k}=\varepsilon^{2} .
$$

As a consequence, $(M, J, \theta)$ is the standard pseudohermitian $(2 n+1)$-sphere $\left(S^{2 n+1}, \widehat{J}, \widehat{\theta}\right)$.

However, regarding (iii) of Remark [1.2, we need an extra condition (1.6) to confirm Conjecture 1.1 in a closed pseudohermitian 3-manifold with nonvanishing torsion.

Theorem 1.4. Let $(M, J, \theta)$ be a closed pseudohermitian 3-manifold with the nonnegative Paneitz operator $P_{0}$. Suppose that

$$
\operatorname{Ric}_{m}(Z, Z)-\operatorname{Tor}_{m}(Z, Z) \geq k\langle Z, Z\rangle_{L_{\theta}},
$$

for all $m \in M, Z \in T_{1,0}$, some positive constant $k$ and

$$
A_{11, \overline{1}}=0 .
$$

Assume that

$$
\lambda_{1}=\frac{k}{2}
$$

and there exists one corresponding eigenfunction $\varphi$ satisfying

$$
P_{1} \varphi=0 \text {. }
$$


Then

$$
d_{\varepsilon}(M)=\sqrt{\frac{2}{k}} \pi \text { with } \quad \frac{2}{k}=\varepsilon^{2} .
$$

As a consequence, $(M, J, \theta)$ is the standard pseudohermitian sphere $\left(S^{3}, \widehat{J}, \widehat{\theta}\right)$.

In particular, it follows from Remark 1.1 and (3.4) that conditions (1.5), (1.3) and (1.6) are satisfied in a closed pseudohermitian $(2 n+1)$-manifold with vanishing torsion. Then we recaptured our previous result for the CR Obata's Theorem ([CC2, [CC3]) as follows:

Corollary 1.5. Let $(M, J, \theta)$ be a closed pseudohermitian $(2 n+1)$-manifold with vanishing torsion. Suppose that

$$
\operatorname{Ric}_{m}(Z, Z) \geq k\langle Z, Z\rangle_{L_{\theta}}
$$

for all $m \in M, Z \in T_{1,0}$ and some positive constant $k$. Assume that

$$
\lambda_{1}=\frac{n k}{n+1} .
$$

Then $(M, J, \theta)$ is the standard pseudohermitian $(2 n+1)$-sphere $\left(S^{2 n+1}, \widehat{J}, \widehat{\theta}\right)$.

\section{Preliminary}

Let us give a brief introduction of pseudohermitian geometry (see Le for more details). Let $(M, \xi)$ be a $(2 n+1)$-dimensional, orientable, contact manifold with contact structure $\xi, \operatorname{dim}_{R} \xi=2 n$. A CR structure compatible with $\xi$ is an endomorphism $J: \xi \rightarrow \xi$ such that $J^{2}=-1$. We also assume that $J$ satisfies the following integrability condition: If $X$ and $Y$ are in $\xi$, then so is $[J X, Y]+[X, J Y]$, and $J([J X, Y]+[X, J Y])=[J X, J Y]-[X, Y]$. A CR structure $J$ can extend to $\mathbb{C} \otimes \xi$ and decomposes $\mathbb{C} \otimes \xi$ into the direct sum of $T_{1,0}$ and $T_{0,1}$, which are eigenspaces of $J$ with respect to $i$ and $-i$, respectively. A manifold $M$ with a CR structure is called a CR manifold. A pseudohermitian structure compatible with $\xi$ is a $C R$ structure $J$ compatible with $\xi$ together with a choice of contact form $\theta$. Such a choice determines a unique real vector field $T$ transverse to $\xi$, which is called the characteristic vector field of $\theta$, such that $\theta(T)=1$ and $\mathcal{L}_{T} \theta=0$ or $d \theta(T, \cdot)=0$. Let $\left\{T, Z_{\alpha}, Z_{\bar{\alpha}}\right\}$ be a frame of $T M \otimes \mathbb{C}$, where $Z_{\alpha}$ is any local frame of $T_{1,0}, Z_{\bar{\alpha}}=\overline{Z_{\alpha}} \in T_{0,1}$ and $T$ is the characteristic vector field. Then $\left\{\theta, \theta^{\alpha}, \theta^{\bar{\alpha}}\right\}$, which is the coframe dual to $\left\{T, Z_{\alpha}, Z_{\bar{\alpha}}\right\}$, satisfies

$$
d \theta=i h_{\alpha \bar{\beta}} \theta^{\alpha} \wedge \theta^{\bar{\beta}}
$$

for some positive definite hermitian matrix of functions $\left(h_{\alpha \bar{\beta}}\right)$.

The Levi form $h=\langle,\rangle_{L_{\theta}}$ is the hermitian form on $T_{1,0}$ defined by

$$
\langle Z, W\rangle_{L_{\theta}}=-i\langle d \theta, Z \wedge \bar{W}\rangle .
$$

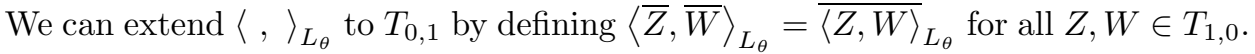
The Levi form naturally induces a hermitian form on the dual bundle of $T_{1,0}$, denoted by $\langle,\rangle_{L_{\theta}^{*}}$, and hence on all the induced tensor bundles.

Moreover, we can define a family of Webster (adapted) Riemannian metrics $g_{\varepsilon}$ of $(M, J, \theta)$ by

$$
g_{\varepsilon}=(1 / 2) d \theta+\varepsilon^{-2} \theta^{2}, \varepsilon>0 .
$$


The pseudohermitian connection of $(J, \theta)$ is the connection $\nabla$ on $T M \otimes \mathbb{C}$ (and extended to tensors) given in terms of a local frame $Z_{\alpha} \in T_{1,0}$ by

$$
\nabla Z_{\alpha}=\theta_{\alpha}{ }^{\beta} \otimes Z_{\beta}, \quad \nabla Z_{\bar{\alpha}}=\theta_{\bar{\alpha}}{ }^{\bar{\beta}} \otimes Z_{\bar{\beta}}, \quad \nabla T=0,
$$

where $\theta_{\alpha}{ }^{\beta}$ are the 1 -forms uniquely determined by the following equations:

$$
\begin{aligned}
d \theta^{\beta} & =\theta^{\alpha} \wedge \theta_{\alpha}{ }^{\beta}+\theta \wedge \tau^{\beta}, \\
0 & =\tau_{\alpha} \wedge \theta^{\alpha}, \\
0 & =\theta_{\alpha}{ }^{\beta}+\theta_{\bar{\beta}}{ }^{\bar{\alpha}} .
\end{aligned}
$$

We can write (by the Cartan lemma) $\tau_{\alpha}=A_{\alpha \gamma} \theta^{\gamma}$ with $A_{\alpha \gamma}=A_{\gamma \alpha}$. The curvature of the Webster-Stanton connection, expressed in terms of the coframe $\{\theta=$ $\left.\theta^{0}, \theta^{\alpha}, \theta^{\bar{\alpha}}\right\}$, is

$$
\begin{aligned}
& \Pi_{\beta}{ }^{\alpha}=\bar{\Pi}_{\bar{\beta}}^{\bar{\alpha}}=d \omega_{\beta}{ }^{\alpha}-\omega_{\beta}{ }^{\gamma} \wedge \omega_{\gamma}{ }^{\alpha}, \\
& \Pi_{0}{ }^{\alpha}=\Pi_{\alpha}{ }^{0}=\Pi_{0}{ }^{\bar{\beta}}=\Pi_{\bar{\beta}}{ }^{0}=\Pi_{0}{ }^{0}=0 .
\end{aligned}
$$

Webster showed that $\Pi_{\beta}{ }^{\alpha}$ can be written as

$$
\Pi_{\beta}{ }^{\alpha}=R_{\beta}{ }^{\alpha}{ }_{\rho \bar{\sigma}} \theta^{\rho} \wedge \theta^{\bar{\sigma}}+W_{\beta}{ }^{\alpha}{ }_{\rho} \theta^{\rho} \wedge \theta-W^{\alpha}{ }_{\beta \bar{\rho}} \theta^{\bar{\rho}} \wedge \theta+i \theta_{\beta} \wedge \tau^{\alpha}-i \tau_{\beta} \wedge \theta^{\alpha},
$$

where the coefficients satisfy

$$
R_{\beta \bar{\alpha} \rho \bar{\sigma}}=\overline{R_{\alpha \bar{\beta} \sigma \bar{\rho}}}=R_{\bar{\alpha} \beta \bar{\sigma} \rho}=R_{\rho \bar{\alpha} \beta \bar{\sigma}}, \quad W_{\beta \bar{\alpha} \gamma}=W_{\gamma \bar{\alpha} \beta} .
$$

We will denote components of covariant derivatives with indices preceded by a comma; thus we write $A_{\alpha \beta, \gamma}$. The indices $\{0, \alpha, \bar{\alpha}\}$ indicate derivatives with respect to $\left\{T, Z_{\alpha}, Z_{\bar{\alpha}}\right\}$. For derivatives of a scalar function, we will often omit the comma, for instance, $f_{\alpha}=Z_{\alpha} f, f_{\alpha \bar{\beta}}=Z_{\bar{\beta}} Z_{\alpha} f-\theta_{\alpha}^{\gamma}\left(Z_{\bar{\beta}}\right) Z_{\gamma} f, f_{0}=T f$ for a (smooth) function.

For a real function $f$, the subgradient $\nabla_{b}$ is defined by $\nabla_{b} f \in \xi$ and $\left\langle Z, \nabla_{b} f\right\rangle_{L_{\theta}}=$ $d f(Z)$ for all vector fields $Z$ tangent to the contact plane. Locally

$$
\nabla_{b} f=\sum_{\alpha} f_{\bar{\alpha}} Z_{\alpha}+f_{\alpha} Z_{\bar{\alpha}}
$$

We can use the connection to define the subhessian as the complex linear map

$$
\left(\nabla^{H}\right)^{2} f: T_{1,0} \oplus T_{0,1} \rightarrow T_{1,0} \oplus T_{0,1}
$$

by

$$
\left(\nabla^{H}\right)^{2} f(Z)=\nabla_{Z} \nabla_{b} f
$$

Also,

$$
\Delta_{b} f=\operatorname{Tr}\left(\left(\nabla^{H}\right)^{2} f\right)=\sum_{\alpha}\left(f_{\alpha \bar{\alpha}}+f_{\bar{\alpha} \alpha}\right) .
$$

The Webster-Ricci tensor and the torsion tensor on $T_{1,0}$ are defined by

$$
\operatorname{Ric}(X, Y)=R_{\alpha \bar{\beta}} X^{\alpha} Y^{\bar{\beta}}
$$

and

$$
\operatorname{Tor}(X, Y)=i \sum_{\alpha, \beta}\left(A_{\bar{\alpha} \bar{\beta}} X^{\bar{\alpha}} Y^{\bar{\beta}}-A_{\alpha \beta} X^{\alpha} Y^{\beta}\right) .
$$

We also define the following tensor $A^{2}$ as

$$
A^{2}(X, Y)=\sum_{\gamma}\left(A_{\alpha \gamma} A_{\bar{\beta} \bar{\gamma}} X^{\alpha} Y^{\bar{\beta}}\right),
$$

where $X=X^{\alpha} Z_{\alpha}, Y=Y^{\beta} Z_{\beta}, R_{\alpha \bar{\beta}}=R_{\gamma}{ }^{\gamma}{ }_{\alpha \bar{\beta}}$. The Webster scalar curvature is $R=R_{\alpha}{ }^{\alpha}=h^{\alpha \bar{\beta}} R_{\alpha \bar{\beta}}$. 


\section{The Diameter estimates}

Let $(M, J, \theta)$ be a closed pseudohermitian $(2 n+1)$-manifold. In this section, we will obtain a lower bound estimate for the diameter of $M$ when a sharp lower estimate for the first positive eigenvalue of the sublaplacian on a pseudohermitian $(2 n+1)$-manifold $M$ is achieved.

First we recall the following CR Bochner formula which involves the CR Paneitz operator.

Lemma 3.1 ([CC2 $)$. Let $(M, J, \theta)$ be a closed pseudohermitian $(2 n+1)$-manifold. For a (smooth) real function $\varphi$ on $M$, we have

$$
\begin{aligned}
\frac{1}{2} \Delta_{b}\left|\nabla_{b} \varphi\right|^{2}= & \left|\left(\nabla^{H}\right)^{2} \varphi\right|^{2}+\left(1+\frac{2}{n}\right)\left\langle\nabla_{b} \varphi, \nabla_{b} \Delta_{b} \varphi\right\rangle_{L_{\theta}} \\
& +[2 \operatorname{Ric}-(n+2) \operatorname{Tor}]\left(\left(\nabla_{b} \varphi\right)_{\mathbb{C}},\left(\nabla_{b} \varphi\right)_{\mathbb{C}}\right) \\
& -\frac{4}{n}\left\langle P \varphi+\bar{P} \varphi, d_{b} \varphi\right\rangle_{L_{\theta}^{*}},
\end{aligned}
$$

where $\left(\nabla_{b} \varphi\right)_{\mathbb{C}}=\sum_{\alpha} \varphi_{\bar{\alpha}} Z_{\alpha}$ is the corresponding complex $(1,0)$-vector field of $\nabla_{b} \varphi$ and $d_{b} \varphi=\varphi_{\alpha} \theta^{\alpha}+\varphi_{\bar{\alpha}} \theta^{\bar{\alpha}}$.

Recall that formula (3.1) follows from the two identities:

$$
\begin{aligned}
\frac{1}{2} \Delta_{b}\left|\nabla_{b} \varphi\right|^{2}= & \left|\left(\nabla^{H}\right)^{2} \varphi\right|^{2}+\left\langle\nabla_{b} \varphi, \nabla_{b} \Delta_{b} \varphi\right\rangle_{L_{\theta}} \\
& +[2 R i c-(n-2) \text { Tor }]\left(\left(\nabla_{b} \varphi\right)_{\mathbb{C}},\left(\nabla_{b} \varphi\right)_{\mathbb{C}}\right) \\
& +2\left\langle J \nabla_{b} \varphi, \nabla_{b} \varphi_{0}\right\rangle_{L_{\theta}}
\end{aligned}
$$

and

$$
\begin{aligned}
n\left\langle J \nabla_{b} \varphi, \nabla_{b} \varphi_{0}\right\rangle_{L_{\theta}}= & \left\langle\nabla_{b} \varphi, \nabla_{b} \Delta_{b} \varphi\right\rangle_{L_{\theta}}-2 n \operatorname{Tor}\left(\left(\nabla_{b} \varphi\right)_{\mathbb{C}},\left(\nabla_{b} \varphi\right)_{\mathbb{C}}\right) \\
& -2\left\langle P \varphi+\bar{P} \varphi, d_{b} \varphi\right\rangle_{L_{\theta}^{*}}
\end{aligned}
$$

We also need the following lemma which follows from an observation as in the proof of the sharp lower bound estimate for the first positive eigenvalue $\lambda_{1}$ of the sublaplacian $\Delta_{b}$ on a closed pseudohermitian 3-manifold in Chi.

Let $\varphi$ be the corresponding eigenfunction of the sublaplacian $\Delta_{b}$ with respect to $\lambda_{1}$. That is, $\Delta_{b} \varphi=-\lambda_{1} \varphi$.

Lemma 3.2. Let $(M, J, \theta)$ be a closed pseudohermitian $(2 n+1)$-manifold. Suppose that

$$
\operatorname{Ric}_{m}(Z, Z)-\frac{n+1}{2} \operatorname{Tor}_{m}(Z, Z) \geq k\langle Z, Z\rangle_{L_{\theta}},
$$

for all $m \in M, Z \in T_{1,0}$, some positive constant $k$ and

$$
\lambda_{1}=\frac{n k}{n+1} .
$$

In addition, we assume that $P_{0}$ is nonnegative if $n=1$. Then the corresponding eigenfunction $\varphi$ will satisfy

$$
P_{0} \varphi=0
$$

and

$$
\varphi_{\alpha \beta}=0, \quad \varphi_{\alpha \bar{\beta}}=0 \text { with } \alpha \neq \beta, \text { and } \varphi_{\alpha \bar{\alpha}}=\varphi_{\beta \bar{\beta}} \text {. }
$$


Moreover, we have

$$
\left[\operatorname{Ric}_{m}-\frac{n+1}{2} \text { Tor }_{m}\right]\left(\left(\nabla_{b} \varphi\right)_{\mathbb{C}},\left(\nabla_{b} \varphi\right)_{\mathbb{C}}\right)=k\left\langle\left(\nabla_{b} \varphi\right)_{\mathbb{C}},\left(\nabla_{b} \varphi\right)_{\mathbb{C}}\right\rangle_{L_{\theta}} .
$$

Proof. By integrating the two identities (3.2) and (3.3), we obtain

$$
\begin{aligned}
\int\left|\left(\nabla^{H}\right)^{2} \varphi\right|^{2} d \mu= & \int\left(\Delta_{b} \varphi\right)^{2} d \mu+2 \int \varphi_{0}^{2} d \mu \\
& -\int[2 \operatorname{Ric}-(n-2) \operatorname{Tor}]\left(\left(\nabla_{b} \varphi\right)_{\mathbb{C}},\left(\nabla_{b} \varphi\right)_{\mathbb{C}}\right) d \mu
\end{aligned}
$$

and

$$
\begin{aligned}
\int \varphi_{0}^{2} d \mu= & \frac{1}{n^{2}} \int\left(\Delta_{b} \varphi\right)^{2} d \mu+\frac{2}{n} \int \operatorname{Tor}\left(\left(\nabla_{b} \varphi\right)_{\mathbb{C}},\left(\nabla_{b} \varphi\right)_{\mathbb{C}}\right) d \mu \\
& -\frac{1}{2 n^{2}} \int P_{0} \varphi \cdot \varphi d \mu,
\end{aligned}
$$

since $\left|\left(\nabla^{H}\right)^{2} \varphi\right|^{2}=2 \sum_{\alpha, \beta}\left|\varphi_{\alpha \beta}\right|^{2}+2 \sum_{\alpha, \beta}\left|\varphi_{\alpha \bar{\beta}}\right|^{2}$. Substituting this on the left-hand side of (3.7) and combining with (3.8), we get

$$
\begin{aligned}
-2 \int \sum_{\alpha, \beta}\left|\varphi_{\alpha \beta}\right|^{2} d \mu \geq & -\frac{n+1}{n} \int\left(\Delta_{b} \varphi\right)^{2} d \mu+\frac{3}{4 n} \int P_{0} \varphi \cdot \varphi d \mu \\
& +\int[2 \operatorname{Ric}-(n+1) \operatorname{Tor}]\left(\left(\nabla_{b} \varphi\right)_{\mathbb{C}},\left(\nabla_{b} \varphi\right)_{\mathbb{C}}\right) d \mu \\
\geq & \left(k-\frac{n+1}{n} \lambda_{1}\right) \int\left|\nabla_{b} \varphi\right|^{2} d \mu,
\end{aligned}
$$

where we have used the Cauchy-Schwarz inequalities

$$
\begin{gathered}
\sum_{\alpha, \beta}\left|\varphi_{\alpha \bar{\beta}}\right|^{2} \geq \sum_{\alpha}\left|\varphi_{\alpha \bar{\alpha}}\right|^{2} \geq \frac{1}{n}\left|\sum_{\alpha} \varphi_{\alpha \bar{\alpha}}\right|^{2}=\frac{1}{4 n}\left(\Delta_{b} \varphi\right)^{2}+\frac{n}{4} \varphi_{0}^{2}, \\
{\left[\operatorname{Ric}-\frac{n+1}{2} \operatorname{Tor}\right]\left(\left(\nabla_{b} \varphi\right)_{\mathbb{C}},\left(\nabla_{b} \varphi\right)_{\mathbb{C}}\right) \geq k\left\langle\left(\nabla_{b} \varphi\right)_{\mathbb{C}},\left(\nabla_{b} \varphi\right)_{\mathbb{C}}\right\rangle_{L_{\theta}},}
\end{gathered}
$$

and

$$
\int P_{0} \varphi \cdot \varphi d \mu \geq 0
$$

It follows that $k-\frac{n+1}{n} \lambda_{1} \leq 0$, i.e. $\lambda_{1} \geq \frac{n k}{n+1}$. In addition, if $\lambda_{1}=\frac{n k}{n+1}$, then all of the above inequalities will become equalities and the results will easily follow.

Lemma 3.3. Let $(M, J, \theta)$ be a closed pseudohermitian $(2 n+1)$-manifold with vanishing torsion. Suppose that

$$
\operatorname{Ric}_{m}(Z, Z) \geq k\langle Z, Z\rangle_{L_{\theta}}
$$

for all $m \in M, Z \in T_{1,0}$ and some positive constant $k$. Then

$$
d_{\varepsilon}(M) \leq \sqrt{\frac{n+1}{k}} \pi \quad \text { with } \frac{n+1}{k}=\varepsilon^{2} .
$$

Proof. Since $\operatorname{Ric}_{m}(Z, Z) \geq k\langle Z, Z\rangle_{L_{\theta}}$, we have ([CC2]

$$
\left(R_{a b}^{\varepsilon}\right)=\left[\begin{array}{lll}
A & B & 0 \\
B^{t} & A & 0 \\
0 & 0 & 0
\end{array}\right]+\left[\begin{array}{cc}
-2 \varepsilon^{-2} I_{2 n \times 2 n} & 0 \\
0 & 2 n \varepsilon^{-2}
\end{array}\right] .
$$


Here $A=\left(2 \operatorname{Re} R_{\alpha \bar{\beta}}\right), B=\left(2 \operatorname{Im} R_{\alpha \bar{\beta}}\right), A+i B=\left[2 R_{\alpha \bar{\beta}}\right]$ and $g_{\varepsilon}=d \theta+\varepsilon^{-2} \theta^{2}$ with $\frac{n+1}{k}=\varepsilon^{2}$. It follows that

$$
\operatorname{Ric}_{g_{\varepsilon}}(X, X) \geq[(2 n+1)-1] \varepsilon^{-2}|X|^{2} .
$$

It follows from Myers' theorem that

$$
d_{\varepsilon}(M) \leq \sqrt{\frac{n+1}{k}} \pi .
$$

Proof of Proposition 1.2. Let $\varphi$ be a nonconstant eigenfunction of the sublaplacian $\Delta_{b}$ satisfying

$$
\Delta_{b} \varphi=-\lambda_{1} \varphi
$$

Consider the smooth function

$$
F=\frac{1}{2}\left|\nabla_{b} \varphi\right|^{2}+\frac{\lambda_{1}}{4 n} \varphi^{2}+\frac{n}{4 \lambda_{1}} \varphi_{0}^{2}
$$

defined on $M$. Computing its sublaplacian

$$
\begin{aligned}
\Delta_{b} F= & \left|\left(\nabla^{H}\right)^{2} \varphi\right|^{2}+\left(1+\frac{2}{n}\right)\left\langle\nabla_{b} \varphi, \nabla_{b} \Delta_{b} \varphi\right\rangle \\
& +[2 \text { Ric }-(n+2) \text { Tor }]\left(\left(\nabla_{b} \varphi\right)_{\mathbb{C}},\left(\nabla_{b} \varphi\right)_{\mathbb{C}}\right) \\
& +\frac{\lambda_{1}}{2 n}\left|\nabla_{b} \varphi\right|^{2}+\frac{\lambda_{1}}{2 n} \varphi \Delta_{b} \varphi+\frac{n}{4 \lambda_{1}} \Delta_{b} \varphi_{0}{ }^{2}
\end{aligned}
$$

where we have used the CR Bochner formula (3.1) with $P_{\beta} \varphi=0$ (by the assumption for $n=1)$ from Lemma 3.2 and Remark 1.1. since $\lambda_{1}=\frac{n k}{n+1}$. On the other hand, due to (3.5) we have ([CTW]

$$
\left|\left(\nabla^{H}\right)^{2} \varphi\right|^{2}=\frac{1}{2 n}\left(\Delta_{b} \varphi\right)^{2}+\frac{n}{2} \varphi_{0}^{2},
$$

and since $\left[\Delta_{b}, T\right]=2 \operatorname{Im} Q$ with the purely holomorphic second-order operator $Q \varphi=2 i\left(A^{\alpha \beta} \varphi_{\alpha}\right), \beta(\mathrm{GL})$

$$
\begin{aligned}
\Delta_{b} \varphi_{0}^{2} & =2 \varphi_{0} \Delta_{b} \varphi_{0}+2\left|\nabla_{b} \varphi_{0}\right|^{2} \\
& =-2 \lambda_{1} \varphi_{0}{ }^{2}+2\left|\nabla_{b} \varphi_{0}\right|^{2}+4 \operatorname{Im}(Q \varphi) \varphi_{0} .
\end{aligned}
$$

Substituting (3.14) and (3.15) into (3.13), one obtains

$$
\begin{aligned}
\Delta_{b} F= & \left(k-\frac{2 n+3}{2 n} \lambda_{1}\right)\left|\nabla_{b} \varphi\right|^{2}-\operatorname{Tor}\left(\left(\nabla_{b} \varphi\right)_{\mathbb{C}},\left(\nabla_{b} \varphi\right)_{\mathbb{C}}\right) \\
& +\frac{n}{2 \lambda_{1}}\left|\nabla_{b} \varphi_{0}\right|^{2}+\frac{n}{\lambda_{1}} \operatorname{Im}(Q \varphi) \varphi_{0}
\end{aligned}
$$

On the other hand, from Lemma 3.2, we have

$$
Q \varphi=0
$$

if $A^{\alpha \beta}, \beta=0$. Hence

$$
\Delta_{b} F=\left(k-\frac{2 n+3}{2 n} \lambda_{1}\right)\left|\nabla_{b} \varphi\right|^{2}-\operatorname{Tor}\left(\left(\nabla_{b} \varphi\right)_{\mathbb{C}},\left(\nabla_{b} \varphi\right)_{\mathbb{C}}\right)+\frac{n}{2 \lambda_{1}}\left|\nabla_{b} \varphi_{0}\right|^{2} .
$$

Next we compute the term $\left|\nabla_{b} \varphi_{0}\right|^{2}$. Since $\lambda_{1}=\frac{n k}{n+1}$, we have

$$
P_{\beta} \varphi=\varphi \bar{\alpha}_{\beta}^{\bar{\alpha}}+i n A_{\beta \alpha} \varphi^{\alpha}=0
$$


for all $\beta$ and

$$
-2 \varphi_{\bar{\alpha}}{ }^{\bar{\alpha}}=\square_{b} \varphi=-\Delta_{b} \varphi+i n \varphi_{0}=\lambda_{1} \varphi+i n \varphi_{0},
$$

thus

$$
i n A_{\beta \alpha} \varphi^{\alpha}=-\varphi_{\bar{\alpha}}{ }^{\bar{\alpha}}{ }=\frac{1}{2}\left(\lambda_{1} \varphi+i n \varphi_{0}\right)_{\beta} .
$$

It follows that

$$
\varphi_{0 \beta}=2 A_{\beta \alpha} \varphi^{\alpha}+i \frac{\lambda_{1}}{n} \varphi_{\beta}
$$

for all $\beta$. Thus

$$
\begin{aligned}
\left|\nabla_{b} \varphi_{0}\right|^{2} & =2 \sum_{\beta} \varphi_{0 \beta} \varphi_{0 \bar{\beta}} \\
& =2 \sum_{\beta}\left(2 A_{\beta \alpha} \varphi^{\alpha}+i \frac{\lambda_{1}}{n} \varphi_{\beta}\right)\left(2 A_{\bar{\beta} \bar{\gamma}} \varphi^{\bar{\gamma}}-i \frac{\lambda_{1}}{n} \varphi_{\bar{\beta}}\right) \\
& =\frac{\lambda_{1}^{2}}{n^{2}}\left|\nabla_{b} \varphi\right|^{2}+\left(\frac{4 \lambda_{1}}{n} \text { Tor }+8 A^{2}\right)\left(\left(\nabla_{b} \varphi\right)_{\mathbb{C}},\left(\nabla_{b} \varphi\right)_{\mathbb{C}}\right) .
\end{aligned}
$$

Substituting (3.18) into (3.16), we conclude that

$$
\begin{aligned}
\Delta_{b} F= & \left(k-\frac{n+1}{n} \lambda_{1}\right)\left|\nabla_{b} \varphi\right|^{2}+\operatorname{Tor}\left(\left(\nabla_{b} \varphi\right)_{\mathbb{C}},\left(\nabla_{b} \varphi\right)_{\mathbb{C}}\right) \\
& +\frac{4 n}{\lambda_{1}} A^{2}\left(\left(\nabla_{b} \varphi\right)_{\mathbb{C}},\left(\nabla_{b} \varphi\right)_{\mathbb{C}}\right) \\
= & {\left[\text { Tor }+\frac{4 n}{\lambda_{1}} A^{2}\right]\left(\left(\nabla_{b} \varphi\right)_{\mathbb{C}},\left(\nabla_{b} \varphi\right)_{\mathbb{C}}\right) } \\
= & {\left[\text { Tor }+\frac{4(n+1)}{k} A^{2}\right]\left(\left(\nabla_{b} \varphi\right)_{\mathbb{C}},\left(\nabla_{b} \varphi\right)_{\mathbb{C}}\right) } \\
\geq & 0 .
\end{aligned}
$$

Since $M$ is closed, $F$ must be identically constant. Moreover,

$$
\frac{1}{2}\left|\nabla_{b} \varphi\right|^{2}+\frac{\lambda_{1}}{4 n} \varphi^{2}+\frac{n}{4 \lambda_{1}} \varphi_{0}^{2}=\frac{\lambda_{1}}{4 n}|\varphi|_{\infty}^{2},
$$

where $|\varphi|_{\infty}=\sup _{M}|\varphi|$. If we normalize $\varphi$ such that $|\varphi|_{\infty}=1$ and observe that at the maximum and minimum points of $\varphi$ its subgradient and derivative with respect to $T$ must vanish, then we obtain that $\max \varphi=1=-\min \varphi$ and so

$$
\left|\nabla_{b} \varphi\right|^{2}+\frac{\lambda_{1}}{2 n} \varphi^{2}+\frac{n}{2 \lambda_{1}} \varphi_{0}^{2}=\frac{\lambda_{1}}{2 n} .
$$

Hence

$$
\frac{k}{2(n+1)}=\frac{\left|\nabla_{b} \varphi\right|^{2}+\frac{n+1}{2 k} \varphi_{0}^{2}}{1-\varphi^{2}} .
$$

Now let $\nabla^{\varepsilon}$ be the gradient with respect to $g_{\varepsilon}$. We have

$$
\left|\nabla^{\varepsilon} \varphi\right|^{2}=2\left|\nabla_{b} \varphi\right|^{2}+\varepsilon^{2} \varphi_{0}^{2}=2\left|\nabla_{b} \varphi\right|^{2}+\frac{n+1}{k} \varphi_{0}^{2}
$$

with $\varepsilon^{2}=\frac{n+1}{k}$. Thus

$$
\left|\nabla_{b} \varphi\right|^{2}+\frac{n+1}{2 k} \varphi_{0}^{2}=\frac{1}{2}\left|\nabla^{\varepsilon} \varphi\right|^{2},
$$


and so

$$
\frac{k}{n+1}=\frac{\left|\nabla^{\varepsilon} \varphi\right|^{2}}{1-\varphi^{2}}
$$

Integrating this along a minimal geodesic $\gamma$ with respect to $g_{\varepsilon}$ joining the points where $\varphi=1$ and $\varphi=-1$, we have

$$
\begin{aligned}
d_{\varepsilon} \sqrt{\frac{k}{n+1}} & \geq \int_{\gamma} \sqrt{\frac{k}{n+1}} d s \\
& =\int_{\gamma} \frac{\left|\nabla^{\varepsilon} \varphi\right|}{\sqrt{1-\varphi^{2}}} d s \\
& \geq \int_{-1}^{1} \frac{d \varphi}{\sqrt{1-\varphi^{2}}} \\
& =\pi,
\end{aligned}
$$

which implies that

$$
d_{\varepsilon} \geq \sqrt{\frac{n+1}{k}} \pi=\varepsilon \pi
$$

Lemma 3.4. Let $(M, J, \theta)$ be a closed pseudohermitian 3-manifold with nonnegative CR Paneitz operator $P_{0}$. Suppose that the curvature condition (1.1) holds, $\lambda_{1}=\frac{k}{2}$ and $A_{\overline{11}}, 1=0$. Let $\varphi$ be the corresponding eigenfunction of the sublaplacian $\Delta_{b}$ with respect to $\lambda_{1}$ and $P_{1} \varphi=0$ on $M$. Then

$$
\left|A_{11}\right|^{2}\left|\nabla_{b} \varphi\right|^{2}=0
$$

on $M$. In particular, the torsion must be vanishing

$$
A_{11}=0
$$

on $(M, J, \theta)$.

Remark 3.1. For generic metrics in a closed Riemannian manifold, all of the eigenfunctions are Morse functions, and consequently their critical point sets are discrete ([U]).

Proof. From Lemma 3.2, we have $P_{0} \varphi=8\left(P_{1} \varphi\right),_{\overline{1}}=0, \varphi_{11}=0$, and thus

$$
\begin{aligned}
\int\left(P_{1} \varphi\right)\left(\overline{P_{1}} \varphi\right) d \mu & =\int\left(\varphi_{\overline{1} 11}+i A_{11} \varphi_{\overline{1}}\right)\left(\varphi_{1 \overline{11}}-i A_{\overline{11}} \varphi_{1}\right) d \mu \\
& =\int\left[-\left(P_{1} \varphi\right)_{, \overline{1}} \varphi_{1 \overline{1}}+\left(i A_{\overline{11}} \varphi_{1}\right)_{, 1} \varphi_{\overline{11}}+\frac{1}{2}\left|A_{11}\right|^{2}\left|\nabla_{b} \varphi\right|^{2}\right] d \mu \\
& =\frac{1}{2} \int\left|A_{11}\right|^{2}\left|\nabla_{b} \varphi\right|^{2} d \mu,
\end{aligned}
$$

which immediately implies the first part of the lemma.

On the other hand, it follows from (3.19) that $A_{11}=0$ on the complement of the critical point set $\Sigma$ of $\varphi$. Then the torsion is vanishing on $(M, J, \theta)$ by continuity property if $\Sigma$ is at most codimension one.

More precisely, $\Sigma_{0}=\Sigma \cap\{p: \varphi(p)=0\}$ is at most of codimension 2 ([HHL]) and $\Sigma \backslash \Sigma_{0}$ is at most of codimension one. In fact, since $\Delta_{b} \varphi=-\lambda_{1} \varphi$, then for $p \in \Sigma \backslash \Sigma_{0}$

$$
\varphi_{1 \overline{1}}(p) \neq 0 \quad \text { and } \quad \varphi_{\overline{11}}(p) \neq 0 .
$$

Define

We have

$$
f=\varphi_{1}+\varphi_{\overline{1}} .
$$

$$
f(p)=0 .
$$


Hence

$$
\Sigma \backslash \Sigma_{0} \subset\{f=0\} .
$$

But from (3.5), for $p \in \Sigma \backslash \Sigma_{0}$,

$$
f_{1}(p)=\varphi_{11}(p)+\varphi_{\overline{1} 1}(p)=\varphi_{\overline{1} 1}(p) \neq 0,
$$

and then

$$
\nabla_{b} f \neq 0
$$

on $\Sigma \backslash \Sigma_{0}$.

It follows that $\Sigma \backslash \Sigma_{0}$ is at most of codimension one. All these imply $\Sigma$ is at most of codimension one.

Lemma 3.5. Let $(M, J, \theta)$ be a closed pseudohermitian $(2 n+1)$-manifold with $n \geq 2$. Suppose that

$$
\operatorname{Ric}_{m}(Z, Z)-\frac{n+1}{2} \operatorname{Tor}_{m}(Z, Z) \geq k\langle Z, Z\rangle_{L_{\theta}},
$$

for all $m \in M, Z \in T_{1,0}$ and

$$
A_{\alpha \gamma, \bar{\gamma}}=0 ; \quad A_{\alpha \beta, \gamma \bar{\gamma}}=0 \quad \text { for all } \alpha, \beta .
$$

Assume that

$$
\lambda_{1}=\frac{n k}{n+1} .
$$

Then

$$
A_{\alpha \beta}=0
$$

on $(M, J, \theta)$.

Proof. From Lemma 3.2, we have $P_{\beta} \varphi=0, P_{0} \varphi=8\left(P_{\beta} \varphi\right)_{\bar{\beta}}=0, \varphi_{\alpha \beta}=0$, and the similar argument

$$
\begin{aligned}
0 & =\int\left(P_{\beta} \varphi\right)\left(\overline{P_{\beta}} \varphi\right) d \mu=\int\left(\varphi_{\bar{\alpha} \alpha \beta}+i n A_{\beta \alpha} \varphi_{\bar{\alpha}}\right)\left(\varphi_{\gamma \bar{\gamma} \bar{\beta}}-i n A_{\bar{\beta} \bar{\gamma}} \varphi_{\gamma}\right) d \mu \\
& =\int\left[-\left(P_{\beta} \varphi\right)_{\bar{\beta}} \varphi_{\gamma \bar{\gamma}}+\left(i n A_{\bar{\beta} \bar{\gamma}} \varphi_{\gamma}\right)_{\beta} \varphi_{\bar{\alpha} \alpha}+n^{2} A_{\beta \alpha} A_{\bar{\beta} \bar{\gamma}} \varphi_{\bar{\alpha}} \varphi_{\gamma}\right] d \mu \\
& =n^{2} \int A_{\beta \alpha} A_{\bar{\beta} \bar{\gamma}} \varphi_{\bar{\alpha}} \varphi_{\gamma} d \mu=n^{2} \int A^{2}\left(\left(\nabla_{b} \varphi\right)_{\mathbb{C}},\left(\nabla_{b} \varphi\right)_{\mathbb{C}}\right) d \mu .
\end{aligned}
$$

Hence from $\varphi_{\alpha \bar{\beta}}=0$ with $\alpha \neq \beta, \varphi_{\alpha \bar{\alpha}}=\varphi_{\beta \bar{\beta}}$ and $A_{\beta \alpha, \bar{\alpha}}=0$, we have

$$
\begin{aligned}
0 & =\int A_{\beta \alpha} A_{\bar{\beta} \bar{\gamma}} \varphi_{\bar{\alpha}} \varphi_{\gamma} d \mu \\
& =-\int A_{\beta \alpha} A_{\bar{\beta} \bar{\gamma}, \bar{\alpha}} \varphi_{\gamma} \varphi d \mu-\int A_{\beta \alpha} A_{\bar{\beta} \bar{\gamma}} \varphi_{\gamma \bar{\alpha}} \varphi d \mu \\
& =-\int A_{\beta \alpha} A_{\bar{\beta} \bar{\gamma}, \bar{\alpha}} \varphi_{\gamma} \varphi d \mu-\int A_{\beta \alpha} A_{\bar{\beta} \bar{\alpha}} \varphi_{\alpha \bar{\alpha}} \varphi d \mu \\
& =-\int A_{\beta \alpha} A_{\bar{\beta} \bar{\gamma}, \bar{\alpha}} \varphi_{\gamma} \varphi d \mu-\int\left|A_{\alpha \beta}\right|^{2} \varphi_{1 \overline{1}} \varphi d \mu .
\end{aligned}
$$

On the other hand, from (3.17) we have

$$
-\varphi_{1 \overline{1}}=\frac{1}{2 n} \lambda_{1} \varphi-\frac{1}{2} i \varphi_{0}
$$


Then

$$
0=-2 \int A_{\beta \alpha} A_{\bar{\beta} \bar{\gamma}, \bar{\alpha}} \varphi_{\gamma} \varphi d \mu+\frac{1}{n} \lambda_{1} \int\left|A_{\alpha \beta}\right|^{2} \varphi^{2} d \mu-i \int\left|A_{\alpha \beta}\right|^{2} \varphi_{0} \varphi d \mu .
$$

We compute, by the commutation relation $A_{\bar{\beta} \bar{\gamma}, \bar{\alpha}}=A_{\bar{\beta} \bar{\alpha}, \bar{\gamma}}$,

$$
\begin{aligned}
& -2 \int A_{\beta \alpha} A_{\bar{\beta} \bar{\gamma}, \bar{\alpha}} \varphi_{\gamma} \varphi d \mu=-\int A_{\beta \alpha} A_{\bar{\beta} \bar{\gamma}, \bar{\alpha}}\left(\varphi^{2}\right)_{\gamma} d \mu \\
& =\int A_{\beta \alpha, \gamma} A_{\bar{\beta} \bar{\gamma}, \bar{\alpha}} \varphi^{2} d \mu+\int A_{\beta \alpha} A_{\bar{\beta} \bar{\gamma}, \bar{\alpha} \gamma} \varphi^{2} d \mu \\
& =\int\left|A_{\beta \alpha, \gamma}\right|^{2} \varphi^{2} d \mu+\int A_{\beta \alpha} A_{\bar{\beta} \bar{\alpha}, \bar{\gamma} \gamma} \varphi^{2} d \mu
\end{aligned}
$$

and substitute it into (3.20):

$$
\begin{aligned}
0= & \frac{1}{n} \lambda_{1} \int\left|A_{\alpha \beta}\right|^{2} \varphi^{2} d \mu+\int\left|A_{\beta \alpha, \gamma}\right|^{2} \varphi^{2} d \mu \\
& +\int A_{\beta \alpha} A_{\bar{\beta} \bar{\alpha}, \bar{\gamma} \gamma} \varphi^{2} d \mu-i \int\left|A_{\alpha \beta}\right|^{2} \varphi_{0} \varphi d \mu .
\end{aligned}
$$

But

$$
A_{\bar{\beta} \bar{\alpha}, \bar{\gamma} \gamma}=0 \quad \text { and } \quad \varphi_{0} \text { is real. }
$$

Thus

$$
\frac{1}{n} \lambda_{1} \int\left|A_{\alpha \beta}\right|^{2} \varphi^{2} d \mu+\int\left|A_{\beta \alpha, \gamma}\right|^{2} \varphi^{2} d \mu=0 .
$$

Hence

$$
\left|A_{\alpha \beta}\right|^{2} \varphi^{2}=0 .
$$

Now apply the same method as in the proof of Lemma 3.4. We have

$$
\left|A_{\alpha \beta}\right|=0
$$

on $(M, J, \theta)$.

Proof of Theorem 1.4. It follows from Lemma 3.4. (3.10) and Proposition 1.2 that

$$
d_{\varepsilon}(M) \geq \sqrt{\frac{2}{k}} \pi
$$

with

$$
\operatorname{Ric}_{g_{\varepsilon}}(X, X) \geq 2 \varepsilon^{-2}|X|^{2}=2 \times \frac{k}{2}|X|^{2}
$$

on $\left(M^{3}, g_{\varepsilon}\right)$. Here $g_{\varepsilon}=d \theta+\varepsilon^{-2} \theta^{2}$ with $\frac{2}{k}=\varepsilon^{2}$. Hence from Lemma 3.4 and Lemma 3.3

$$
d_{\varepsilon}(M) \leq \sqrt{\frac{2}{k}} \pi
$$

All these imply

$$
d_{\varepsilon}(M)=\sqrt{\frac{2}{k}} \pi
$$

By applying S.-Y. Cheng's theorem $([\mathrm{Ch}])$, it follows that $(M, J, \theta)$ is the standard pseudohermitian sphere $\left(S^{3}, \widehat{J}, \widehat{\theta}\right)$.

Proof of Theorem 1.3. It follows from Proposition 1.2 Lemma 3.5 and Lemma 3.3 . 


\section{ACKNOWLEDGMENTS}

The first author would like to express his thanks to Professor Jiaping Wang for pointing out the relation between Riemannian diameter estimate and Obata's theorem.

\section{REFERENCES}

[BD] E. Barletta and S. Dragomir, Jacobi fields of the Tanaka-Webster connection on Sasakian manifolds, Kodai Math. J. 29 (2006), no. 3, 406-454. MR2278775(2007h:53060)

[CCC] S.-C. Chang, J.-H. Cheng and H.-L. Chiu, The fourth-order Q-curvature flow on a CR 3-manifold, Indiana Univ. Math. J. 56 (2007), no. 4, 1793-1826. MR2354700|(2009a:53111)

[CC1] S.-C. Chang and H.-L. Chiu, On the estimate of the first eigenvalue of a sublaplacian on a pseudohermitian 3-manifold, Pacific J. Math 232 (2007), no. 2, 269-282. MR 2366354 (2008m:58065)

[CC2] S.-C. Chang and H.-L. Chiu, Nonnegativity of CR Paneitz operator and its Application to the CR Obata's Theorem in a Pseudohermitian $(2 n+1)$-Manifold, J. Geom. Analysis, 19 (2009), 261-287. MR2481962 (2010f:32034)

[CC3] S.-C. Chang and H.-L. Chiu, On the CR Analogue of Obata's Theorem in a Pseudohermitian 3-Manifold, Math. Ann. 345 (2009), 33-51. MR2520051 (2010i:32036)

[Ch] S. Y. Cheng, Eigenvalue Comparison theorems and its Geometric Application, Math. Z. 143 (1975), 289-297. MR0378001 (51:14170)

[Chi] H.-L. Chiu, The sharp lower bound for the first positive eigenvalue of the sublaplacian on a pseudohermitian 3-manifold, AGAG 30 (2006), no. 1, 81-96. MR:2249615 (2007j:58034)

[Cho] W.-L. Chow, Uber System Von Lineaaren Partiellen Differentialgleichungen erster Orduung,. Math. Ann. 117 (1939), 98-105. MR0001880(1:313d)

[CTW] S.-C. Chang, Jingzhu Tie and C.-T. Wu, Subgradient Estimate and Liouville-type Theorem for the CR Heat Equation on Heisenberg groups $\mathbf{H}^{n}$, Asian J. Math. 14 (2010), no. 1, 41-72. MR 2726594

[Ge] H. Geiges, Normal Contact Structures on 3-Manifolds, Tohoku Math. J. 49 (1997), 415422. MR:1464186 (98h:53046)

[GL] C. R. Graham and J. M. Lee, Smooth Solutions of Degenerate Laplacians on Strictly Pseudoconvex Domains, Duke Math. J. 57 (1988), 697-720. MR.975118 (90c:32031)

[Gr] A. Greenleaf, The first eigenvalue of a sublaplacian on a pseudohermitian manifold, Commum. Partial Differential Equations 10 (1985), no. 2,3, 191-217. MR777049 (86f:58157)

$[\mathrm{FH}] \quad$ C. Fefferman and K. Hirachi, Ambient Metric Construction of $Q$-Curvature in Conformal and $C R$ Geometries, Math. Res. Lett. 10, No. 5-6 (2003), 819-831. MR2025058 (2005d:53044)

[HHL] Q. Han, R. Hardt, and F. H. Lin, Geometric Measure of Singular Sets of Elliptic Equations, Comm. Pure Appl. Math. 51 (1998), no. 11-12, 1425-1443. MR.1639155 (99h:35032a)

[Hi] K. Hirachi, Scalar Pseudo-hermitian Invariants and the Szegö Kernel on 3-dimensional CR Manifolds, Lecture Notes in Pure and Appl. Math. 143, Dekker (1992), 67-76. MR.1201602 (93k:32036)

[KT] Y. Kamishima and T. Tsuboi, CR-Structures on Seifert Manifolds, Invent. Math. 104 (1991), 149-163. MR1094049 (92c:53016)

[L] A. Lichnerowicz, Géometrie des groupes de transformations, Dunod, Pairs, 1958.

[Le] J. M. Lee, Pseudo-Einstein Structure on CR Manifolds, Amer. J. Math. 110 (1988), 157178. MR 926742 (89f:32034)

[Li] P. Li, Lecture notes on Geometric Analysis, 1996.

[LL] S.-Y. Li and H.-S. Luk, The sharp lower bound for the first positive eigenvalue of a sublaplacian on a pseudohermitian manifold, Proc. Am. Math. Soc. 132 (2004), no. 3, 789-798. MR2019957 (2005c:58056)

[LY] P. Li and S.-T. Yau, On the Parabolic Kernel of the Schrődinger Operator, Acta Math. 156 (1985), 153-201. MR834612 (87f:58156)

[O] M. Obata, Certain conditions for a Riemannian manifold to be isometric with a sphere, J. Math. Soc. Japan 14 (1962), 333-340. MR0142086 (25:5479)

[Ru] Michel Rumin, Formes Differentielles sur les Varietes de Contact, JDG 39 (1994), 281-330. MR:1267892(95g:58221) 
[U] K. Uhlenbeck, Generic properties of eigenfunctions, Amer. J. Math. 98 (1976), no. 4, 1059-1078. MR0464332 (57:4264)

[W] J.-P. Wang, Tsing-Hua Lecture Note, Hsinchu, Taiwan, 2005.

Department of Mathematics, National Taiwan University, Taipei 10617, Taiwan, Republic of China - and - Taida Institute for Mathematical Sciences (TIMS), National TAiwan University, TAipei 10617, Taiwan, Republic of China

E-mail address: scchang@math.ntu.edu.tw

Department of Applied Mathematics, National PingTung University of Education, PingTung, Taiwan 90003, Republic of China

E-mail address: ctwu@mail.npue.edu.tw 